\title{
Preventive Generation Maintenance Scheduling - a Simulated Annealing Approach to use in Competitive Markets
}

\author{
J. T. Saraiva, Member IEEE, M. L. Pereira, V. T. Mendes, J. C. Sousa
}

\begin{abstract}
The scheduling of preventive maintenance actions of generators is not a new problem but gained in recent years a new interest with the advent of electricity markets. In this paper we report the research on this topic developed during the preparation of the MSc Thesis of the second author. In this paper we formulate the problem as a mixed integer optimization problem and we describe the use of Simulated Annealing to solve it. Simulated Annealing is a very appealing metaheuristic easily implemented and providing good results in numerous optimization problems. The paper includes results obtained for a Case Study based on a realistic generation system. This research work was proposed and developed with the collaboration of the third and fourth authors, from EDP Produção, Portugal.
\end{abstract}

Index Terms-- preventive maintenance, generators, scheduling, Simulated Annealing.

\section{INTRODUCTION}

$\mathrm{T}$ The scheduling of preventive maintenance actions of thermal generators has been subject of study and analysis by many researchers. This implicitly recognizes the importance of this topic in the past in the sense that this was considered a complex problem, whose solution affected the daily unit commitment and the dispatch of the generation systems. The problem had a variety of formulations and integrated a number of variables and constraints, reflecting different levels of refinements that were progressively introduced. Nevertheless, a number of features were common to all these formulations: one aimed at scheduling the maintenance actions of a set of generators along a period of typically one or two years discretized in weeks, ensuring that the expected demand was supplied, that the maintenance period of each generator was continuous in time, that the number of maintenance crews available for each generation technology was not exceeded and that at least one maintenance action was scheduled for each generator along the period under analysis. Typically this corresponded to a combinatorial problem formulated using binary variables having the value 1 if a particular generator was scheduled for maintenance in a particular week. The objective of this problem was usually the minimization of the generation cost along the planning period.

João Tomé Saraiva (jsaraiva@fe.up.pt) is with FEUP, Faculdade de Engenharia da Universidade do Porto and INESC Porto - Instituto de Engenharia de Sistemas e Computadores do Porto, Campus da FEUP, Rua Dr. Roberto Frias, 4200-465 Porto Portugal. Marcelo Leandro Pereira (ee03157@fe.up.pt) completed is MSc in FEUP, Faculdade de Engenharia da Universidade do Porto in March 2009.

Virgílio Torrado Mendes (virgilio.mendes@edp.pt) and José Carlos Sousa (jose.sousa@edp.pt) are with EDP Produção SA, Rua de Sá da Bandeira 567, 4000-437 Porto. José Carlos Sousa completed his MSc in FEUP in June 2005 and is currently a PhD student at FEUP.
With the introduction of market mechanisms in the electricity sector, this problem started to receive increased attention given the implications that the adoption of a good preventive maintenance schedule can have in the revenues of generation agents. In this scope, the Preventive Maintenance Scheduling, PMS, problem gained new dimensions given that it is now strongly affected by uncertainties namely determining the behaviour of the demand, by the presence of renewable generation, several of them dependent on volatile primary resources, and also being dependent on electricity prices now determined by market mechanisms.

In this context, this paper describes a PMS model developed in the scope of the MSc thesis concluded in July 2009 by the second author and in close cooperation with a Portuguese generation company. The model aims at minimizing the cost of generating electricity to meet the demand along the period of one year discretized in 52 weeks, considering minimum and maximum limits of generation units, as well as several other constraints directly related with the maintenance problem. The problem includes binary variables to model a generator being scheduled for maintenance in a particular week. In order to solve this combinatorial problem it was used a well known meta-heuristic, Simulated Annealing that proved to be very efficient in several tests that were performed. This paper includes a Case Study considering a generation system integrating 29 generators and expected values both for weekly values of the demand and of the market prices of electricity.

According to these ideas this paper is structured as follows. Section II summarizes some models and approaches available in the literature addressing the generator preventive maintenance problem. Section III describes the optimization problem and Section IV details the application of Simulated Annealing to solve it. Finally, Section V presents the Case Study and Section VI draws the most relevant conclusions.

\section{GENERATOR PREVENTIVE MAINTENANCE APPROACHES}

The definition of schedules to implement preventive maintenance actions on generation units has been addressed in the past as it is recognized by the number of papers on this topic. These formulations can be grouped in two large sets: the first one including approaches using traditional optimisation techniques and the second adopting metaheuristics to address the integer nature of several variables.

Regarding the first group, reference [1] formulates the optimization problem to identify the most adequate maintenance schedule using an objective function that aggregates 5 terms - the expected energy generation cost along the period under analysis, the maintenance cost, a reliability 
driven term, a term related with deviations of the maintenance program regarding the ideal frequency to develop maintenance actions and, finally, a term related with penalties for constraints that are not fully enforced. On the other hand, in [2] the generator maintenance problem is formulated as a large-scale mixed integer non-linear optimization problem and the author discusses the impact on the solution of relaxing several constraints as well as the integer nature of some variables. In this paper the author adopts a combined implicit enumeration and branch-and-bound algorithm. A similar formulation is described in [3] considering the generation cost, a reliability index and penalties for violated constraints.

Reference [4] discusses the benefits of developing optimal maintenance generator schedules given that sub-optimal programs lead to higher generation costs and lower reliability of the generation system and of the entire power system. Apart from that, the maintenance programs affect short and longterm operation and planning actions as unit commitment, pumping and hydro scheduling. In the developed approach the authors use two optimization criteria - generation cost and reliability, and the formulation uses integer variables $x_{i k}$ that if equal to 1 indicate that the maintenance period of unit $i$ starts at week $\mathrm{k}$. The developed approach uses a probabilistic production cost algorithm based on cumulants in order to get the generation cost for the period under analysis.

Reference [5] proposes a more complex formulation considering a longer planning period and network constraints as well as generator outages. Given the complexity of the resulting problem, the authors use Benders Decomposition to consider network constraints in each planning sub-period.

Regarding the second group, there are several papers using Simulated Annealing, Genetic Algorithms, Tabu Search and fuzzy models to consider different particular aspects of the problem. In this scope, [6] formulates the generator scheduling maintenance problem in a similar way regarding [4] considering an objective function that includes two terms (generation cost and maintenance cost) and constraints related with the continuity of the maintenance actions once started, with the availability of crews to develop maintenance actions for a given generator technology, with specified sequences of maintenance actions for some units, with the generator output limits and with the supply of the demand along the period. This mixed integer problem is solved using Simulated Annealing and the scheduling programs obtained with this approach and with an Integer Programming traditional technique are compared considering a small, a medium and a large generation system. The Simulated Annealing based approach provides faster solutions for the small and medium systems with comparable costs. For the larger generation system the Integer Programming approach is not able to get a solution while the Simulated Annealing provides one.

References [7, 8, 9] describe the use of Genetic Algorithms, combined with Simulated Annealing in case of $[8,9]$. The authors implemented genetic operators to prevent the premature convergence of the simulation together with efficient encoding/decoding techniques concluding that GA's are very effective in dealing with the PMS problem.
The approach described in [10] uses Genetic Algorithms together with fuzzy membership functions to model the two objectives included in this formulation - the reserve margin and the generation cost. Regarding the constraints, this formulation considers limitations on the number of available maintenance crews, limitations on the number of generators in maintenance in the same geographical area in order to limit power transfers between areas and the definition of a window of weeks during which each generator maintenance should be scheduled. Reference [11] also uses a Genetic Algorithm combined with a fuzzy function to evaluate the solutions. This function combines a crisp penalty function to model the inflexible demand constraint together with fuzzy penalty functions to model the objective and other constraints. In [12] it is described a fuzzy approach that is able to deal with uncertainties affecting the demand and the generation and maintenance costs. This approach uses triangular fuzzy numbers to model the demand and an evolutionary algorithm.

In $[13,14]$ the PMS problem is solved using Tabu Search. In [13] it is used a multi-stage approach to decompose the problem in several sub-problems. The partial results are then combined to produce the global maintenance schedule. In [14] the formulation uses the generation cost and the reserve margin as objectives and the constraints are related with the availability of crews, predefined sequence of maintenance actions for several units and continuity of the maintenance period once a maintenance action starts. The plans provided by the Tabu Search algorithm for two generation systems (one with 4 units and another with 22 units) were compared with the results obtained with an implicit enumeration approach. The results obtained with Tabu Search were very promising given the more reduced computation time and their good quality.

References $[15,16]$ compare the performance of several metaheuristic approaches, namely Tabu Search, Simulated Annealing, Genetic Algorithms, an hybrid Simulated Annealing/Genetic Algorithm approach and an hybrid Tabu Search/Simulated Annealing algorithm. The authors report that the combined use of Simulated Annealing/Genetic Algorithm and of Tabu Search/Simulated Annealing produces better results than the isolated use of a single metaheuristic, although the computational time is sometimes longer.

Finally, in [17] the PMS problem is formulated as a dynamic non-cooperative game and the players aim at maximizing their profits coming from selling electricity in the market. The solution corresponds to a Nash equilibrium that is obtained using a backward induction scheme.

\section{MATHEMATICAL FORMULATION}

\section{A. General Ideas}

The developed model considers that thermal stations are either available or completely unavailable due to an outage. Regarding maintenance, the units are either completely in maintenance or not in maintenance. When available, this means the maximum output of each unit is constant. Regarding the constraints, the formulation considers the following ones:

- the maintenance action is performed continuously. This 
means that when started, an unit is unavailable for maintenance for a number of weeks corresponding to the duration of the action;

- each unit should be submitted to one maintenance action per year;

- the demand should always be satisfied;

- there is a limited number of crews to implement maintenance actions for each generation technology.

Considering these general ideas, the formulation aims at minimizing the generation cost along a planning period $\mathrm{T}$, discretized in 52 weeks. The week demand is represented by a diagram organized in 5 steps as follows:

- Step $1-5 \%$ of the week, corresponding to 8,4 hours;

- Step 2-30\% of the week, corresponding to 50,4 hours;

- Step 3-18\% of the week, corresponding to 30,24 hours;

- Step 4-20\% of the week, corresponding to 33,6 hours;

- Step 5-27\% of the week, corresponding to 45,36 hours.

Finally, the approach was developed to schedule maintenance actions of thermal units. In case the system has an hydro component and/or renewable and dispersed generation not submitted to dispatch and paid according to feed-in schemes, it should be estimated the demand to be supplied by the thermal sub-system subtracting the hydro and dispersed generation components from the total demand for each period.

\section{B. Optimization Problem}

Given the above general aspects, we used the following notation to formulate the optimisation problem:

- $C_{k j}$ - generation cost of unit $\mathrm{k}$, in step $\mathrm{j}$;

- $P_{k j t}$ - generation of unit $\mathrm{k}$, in step $\mathrm{j}$, in week $\mathrm{t}$;

- $\Delta_{j}$ - duration in hours of step j;

- $D_{j t}$ - demand in step $\mathrm{j}$, in week t;

- $H_{j t}$ - hydro generation in step $\mathrm{j}$, in week $\mathrm{t}$;

- $W_{j t}$ - wind generation in step $\mathrm{j}$, in week $\mathrm{t}$;

- $O G_{j t}$ - other generation in step j, in week t;

- $I N T_{j t}$ - power from interconnections with other countries in step $\mathrm{j}$, in week $\mathrm{t}$;

- $P_{k j t}^{+}$and $P_{k j t}^{-}$- maximum and minimum powers for unit $\mathrm{k}$, in step $\mathrm{j}$, in week $\mathrm{t}$;

- $m_{k t}$ - state of unit $\mathrm{k}$ in week $\mathrm{t}$ regarding maintenance. If $m_{k t}=1$ unit $\mathrm{k}$ is in maintenance in week t. If $m_{k t}=0$ then it is not in maintenance;

- $i_{k t}$ - operation state of unit $\mathrm{k}$, in week $\mathrm{t}$;

- $f_{k t}$ - state of unit $\mathrm{k}$ in week $\mathrm{t}$ regarding forced outages;

- $P_{k}$ - rated power of unit k;

- $a_{k j}$ - availability of unit $\mathrm{k}$ in step $\mathrm{j}$;

- $S_{k}$ - duration in weeks of the maintenance action of unit k;

- $t_{k}$ - week in which the maintenance action of unit $\mathrm{k}$ will start;

- $t_{k}^{-}$to $t_{k}^{+}$- initial and final weeks of the period during which the maintenance action of unit $\mathrm{k}$ should be initiated;
- $V_{r}^{+}$- maximum number of units of the same technology $\mathrm{r}$ that can be in maintenance simultaneously;

- $V_{r}$ - set of units of the same technology r;

- K - number of units;

- $\mathbf{J}$ - number of steps used to model the demand;

- T - total number of weeks;

- R - number of different thermal generator technologies.

According to this notation the generator maintenance scheduling problem is formulated as follows:

$\min Z=\sum_{t=1}^{T} \sum_{j=1}^{J} \sum_{k=1}^{K} C_{k j}\left(P_{k j t}\right) \cdot \Delta_{j}$

subj. $\quad \sum_{k=1}^{K} P_{k j t}=D_{j t}-H_{j t}-W_{j t}-O G_{j t}-I N T_{j t}$

$$
\text { for } \mathrm{j}=1 \ldots \mathrm{J} ; \mathrm{t}=1 \ldots \mathrm{T}
$$

$$
P_{k j t}^{-} \leq P_{k j t} \leq P_{k j t}^{+} \quad \text { for } \mathrm{k}=1 \ldots \mathrm{K} ; \mathrm{j}=1 \ldots \mathrm{J} ; \mathrm{t}=1 \ldots \mathrm{T}
$$

$P_{k j t}^{+}=\left(1-m_{k t}\right) \cdot i_{k t} \cdot\left(1-f_{k t}\right) \cdot P_{k} \cdot a_{k j}$

$$
\begin{array}{ll}
\sum_{t=1}^{T} m_{k t}=S_{k} & \text { for } \mathrm{k}=1 \ldots \mathrm{K} ; \mathrm{j}=1 \ldots \mathrm{J} ; \mathrm{t}=1 \ldots \mathrm{T} \\
\sum_{t=t_{k}}^{t_{k}+S_{k}-1} m_{k t}=S_{k} & \text { for } \mathrm{k}=1 \ldots \mathrm{K} \\
t_{k}^{-} \leq t_{k} \leq t_{k}^{+} & \text {for } \mathrm{k}=1 \ldots \mathrm{K} \\
t_{k}^{+}-t_{k}^{-}+1 \geq S_{k} & \text { for } \mathrm{k}=1 \ldots \mathrm{K} \\
\sum_{k \in V r} m_{k t} \leq V_{r}^{+} & \text {for } \mathrm{r}=1 \ldots \mathrm{R} \\
m_{k t} \in\{0,1\} & \text { for } \mathrm{k}=1 \ldots \mathrm{K} ; \mathrm{t}=1 \ldots \mathrm{T}
\end{array}
$$

In this formulation, we aim at minimizing the generation cost to supply the demand along the period T. As mentioned before, this period $\mathrm{T}$ is organized in weeks and the demand is specified by a load diagram organized in $\mathbf{J}$ steps. This objective function is subjected to the following constraints:

- constraints (2) enforce that the demand $D_{j t}$ is supplied in each step $\mathrm{j}$ and in every week $\mathrm{t}$. This supply is obtained using the $K$ generation units and also by hydro generation, $H_{j t}$, by wind parks, $W_{j t}$, by other generation sources, $O G_{j t}$, and by interconnections with other countries, INT $j$;

- constraints (3) bound the output of the $K$ units to minimum and maximum values, in each step $j$ and in each week $t$;

- constraints (4) determine the maximum output of unit $k$, in each step $j$ and week $t$. This maximum output depends on the rated power of the unit, $P_{k}$, on its availability regarding maintenance modeled by the variable $m_{k t}$, on its availability regarding forced outages modeled by $i_{k t}$ and on the availability of unit $\mathrm{k}$ in step $j$ modeled by $a_{k j}$;

- constraints (5) enforce that each unit $k$ has to be subjected to a maintenance action per year with a duration of $S_{k}$ weeks; 
- constraints (6) enforce that the $S_{k}$ weeks during which unit $k$ is in maintenance are continuous;

- constraints (7) indicate that the maintenance action of unit $k$ has be initiated between weeks $t_{\bar{k}}$ and $t_{k}^{+}$;

- constraints (8) enforce that that the maintenance action of unit $k$ has to be finished before $t_{k}^{+}$;

- constraints (9) limit the number of simultaneous maintenance actions of units of the same technology $r$, given the available number of crews for technology $r$;

- finally, (10) indicates that variables $m_{k t}$ are binary ones.

\section{Simulated ANNEALING - AN OVERVIEW}

In the last decade, several optimization techniques emerged both in conceptual terms and in current applications. These techniques, often called meta-heuristics, include Tabu Search, Neural Networks, Simulated Annealing, Genetic Algorithms and its development to Genetic Programming. Literature includes nowadays a large number of papers reporting applications of these techniques to several problems showing their success and their special ability to address problems having some particular characteristics.

In particular, Simulated Annealing and Genetic Algorithms are used to address combinatorial problems due to the presence of discrete variables. Traditionally, this type of problems could be tackled in a two-step approach. In a first phase, discrete variables were relaxed into continuous ones, and then the output was rounded to the nearest integer. As it is easily understood, this does not ensure that the selected integer solution corresponds to the optimal one. Other approaches adopted branch-and-bound based techniques, usually leading to a large amount of computation time.

However, there are two aspects that must be mentioned:

- several continuous optimization algorithms - as gradient techniques - often converge to local optima as illustrated in Figure 1. Gradient-based approaches can converge to solution A and the iterative process will be trapped there since derivatives are zero. Apart from that, the final solution can vary depending on initialization conditions;

- secondly, in several real life problems, decision makers are not really interested in the global optimum. They are, in fact, interested in a good or adequate solution, for which some quality index is evaluated. The process would end if an improvement, although not impossible to obtain, can lead to a large computational time.

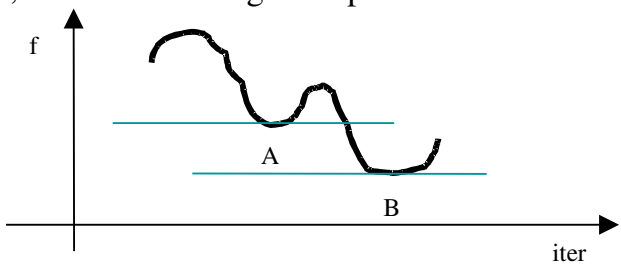

Fig. 1. Illustration of an optimization process with local optima.

Simulated Annealing was developed by Kirkpatrick et al $[18,19]$ based on the Metropolis algorithm dated from 1953. It is a search procedure in which it is included the possibility of accepting a solution that is worse than the current one. The simulation starts at an initial solution, $x_{1}$, evaluates it using an evaluation function, $f\left(x_{1}\right)$, and samples a new solution in the neighborhood of $x_{1}$. If this new solution improves $f\left(x_{1}\right)$, then it is accepted. If it is worse than the current one, it can still be accepted depending on a so-called probability of accepting worse solutions. This mechanism eventually allows escaping from a local optimum, as point $\mathrm{A}$ in Figure 1, and go on iterating towards point B. In a more formal way, the algorithm can be summarized as indicated in the next paragraphs admitting a minimization problem.

\section{Simulated Annealing Basic Algorithm}

i) Initialization: Select an initial solution $x_{1}$ in the solution space X. Evaluate $x_{1}, f\left(x_{1}\right)$;

ii) Assign $x_{1}$ to $x^{*}$ and $f\left(x_{1}\right)$ to $f\left(x^{*}\right)$. The sign $*$ denotes the best solution identified until this step;

iii) Step $\mathrm{n}=1,2, \ldots, \mathrm{n} . x_{n}$ denotes the current solution at iteration $\mathrm{n}$. Obtain a new solution $x$ in the neighborhood of $x_{n}$ using a sampling process;

iv) Testing:

$$
\begin{aligned}
& \text { a.if } f(x) \leq f\left(x_{n}\right) \text { then assign } x \text { to } x_{n+1} ; \\
& \text { if } f(x) \leq f\left(x^{*}\right) \text { then assign } x \text { to } x^{*} \text { and } f(x) \text { to } f\left(x^{*}\right) \text {; }
\end{aligned}
$$

b. else

i. get a random number $\mathrm{p}$ in $[0.0 ; 1.0]$;

ii. evaluate the probability of accepting worse solutions at iteration $\mathrm{n}, p(n)$, using (11);

$$
p(n)=e^{\frac{f\left(x_{n}\right)-f(x)}{K_{\text {Boltz }} \cdot \text { Temperature }}}
$$

if $p \leq p(n)$ then assign $x$ to $x_{n+1}$;

v) End if a stopping rule is reached. Otherwise go to iii).

In expression (11), $K_{\text {Boltz }}$ represents the Boltzman constant. Regarding this algorithm there are some issues to be clarified:

- the solution of a combinatorial problem, $\mathrm{CP}$, has a clear analogy with the cooling process of a thermodynamic system, TDS. In this analogy, a state of a TDS is equivalent to the solutions or combinations of a CP. The energy of a TDS corresponds to the evaluation function, $\mathrm{f}$, of the $\mathrm{CP}$ and the temperature of a TDS corresponds to the control parameter of the $\mathrm{CP}$ problem;

- a TDS system should be cooled in a slow way. This enables sub-systems to reorganize themselves so that a low energy system is built. Similarly, the temperature of the CP must be lowered in a sufficiently slow way in order to identify a good quality solution;

- the temperature $\mathrm{T}$ is usually lowered by steps corresponding to a maximum number of iterations. Once this maximum is reached, the current temperature is lowered by a cooling parameter $\alpha$, in $[0.0 ; 1.0]$. According to this scheme, at the beginning of the simulation, the probability of accepting worse solutions, $p(n)$, is larger. This turns it more probable to accept worse solutions making the search more chaotic in the sense that larger areas of the solution space are searched. As the process goes on, the temperature is lowered, turning it more 
difficult to accept worse solutions. This means that the search is eventually being conducted in a promising area from where one doesn't want to leave;

- the Simulated Annealing algorithm proceeds from one solution $x$ to another one in its neighbourhood. The definition of the neighbourhood of $x, N(x)$, is a strategic aspect of the algorithm in the sense it has an impact on the design of the final solution. The structure of $N(x)$ is quite simple to define in discrete problems. As an example, Simulated Annealing can be used to minimize transmission losses in a network by changing taps of transformers or of capacitor banks. Departing from the nominal positions, one can simply sample a transformer or capacitor, and then sample if the tap goes upwards or downwards by one step. This leads to a neighbour solution regarding the current one;

- finally, the search procedure ends if a stopping rule is achieved. This can correspond to the absence of improvements in a pre-specified number of iterations, to perform a maximum number of iterations or to lower the temperature parameter till a minimum level.

\section{SOLUTION ALGORITHM}

The discrete nature of the PMS problem justified the adoption of Simulated Annealing given its natural adaptation to incorporate discrete variables and parameters and its implementation easiness. This metaheuristic starts at an initial maintenance schedule and evolves to a new schedule by sampling new maintenance periods for some units. Each schedule is evaluated considering the generation costs along the whole period $\mathrm{T}$, plus penalties on violated constraints. A new solution is then identified in the neighborhood of the current one and after evaluating this new solution, a decision is taken to accept it or not. The following paragraphs detail the application of Simulated Annealing to this particular problem.

i) Consider an initial maintenance schedule, denoted by $x^{o}$;

ii) Analyze the current solution:

a. compute the generation cost along the period $T$;

b. check if any constraints (2) to (9) is violated;

c. the value of the Evaluation Function $E V^{o}$ is the sum of the generation cost plus penalties on violated constraints;

d. assign $E V^{o}$ to $E V^{o p t}$ and to $E V^{\text {current }}$;

e. assign $x^{o}$ to $x^{\text {opt }}$ and to $x^{\text {current }}$;

f. set the iteration counter, $I C$, to 1 ;

g. set the worse solution counter, WSC, at 0;

iii) Identify a new schedule. Sample an unit $\mathrm{k}$ and sample a week between $t_{k}^{-}$and $t_{k}^{+}$to start its maintenance. The new schedule is denoted as $x^{\text {new }}$;

iv) Analyze the new schedule computing the generation cost and looking for violated constraints. Obtain $E V^{\text {new }}$;

v) If $E V^{\text {new }}<E V^{\text {opt }}$ then
a. assign $E F^{\text {new }}$ to $E F^{\text {opt }}$ and to $E F^{\text {current }}$;
b. assign $\mathrm{x}^{\text {new }}$ to $\mathrm{x}^{\text {opt }}$ and to $\mathrm{x}^{\text {current }}$;
c. set the worse solution counter, WSC, at 0;

vi) If $E F^{\text {new }} \geq E F^{\text {opt }}$ then

a. get a random number $p \in[0.0 ; 1.0]$;

b. compute the probability of accepting worse solutions $\mathrm{p}\left(\mathrm{x}^{\text {new }}\right)$ by (12);

$p\left(x^{\text {new }}\right)=e^{\frac{E F^{\text {current }}-E F^{\text {new }}}{K_{\text {Boltz }} T}}$

c. if $p \leq p\left(x^{\text {new }}\right)$ then assign $x^{\text {new }}$ to $x^{\text {current }}$ and $E F^{\text {new }}$ to $E F^{\text {current }}$;

d. increase the worse solution counter, WSC, by 1 ;

vii)If WSC is larger than a specified maximum number of iterations without improvements than go to ix);

viii) If the iteration counter $I C$ is larger than the maximum number of iterations per temperature level then:

a. decrease the temperature level $\mathrm{T}$ by a rate $\alpha$ smaller then 1.0;

b. if the new temperature level is smaller then the minimum allowed temperature then go to ix);

c. set the iteration counter $I C$ to 1 ;

Else, increase the iteration counter $I C$ by 1 ; Go back to iii);

ix) End.

\section{CASE STUDY}

\section{A. Data}

The generation test system includes 29 thermal units as follows:

- coal fire plants $-2360 \mathrm{MW}$ in 8 units;

- CCGT - 3006 MW in 8 units;

- fuel oil - 1708 MW in 11 units;

- diesel - $165 \mathrm{MW}$ in 2 units.

Apart from these units, the generation system also has inputs from hydro stations, wind parks, other generation sources (as cogeneration and small hydro stations dispersed along the network) and interconnections with other countries. In order to incorporate a reliability index in the model, we also considered a fictitious station to model Energy Not Supplied. This station has a very large generation cost when compared with the costs of the other thermal stations. This means that, in a given week $\mathrm{t}$ and demand step $\mathrm{j}$, it will be dispatched only if the other cheaper stations are already in the limit. This implicitly means that the corresponding maintenance schedule has a large concentration of units simultaneously in maintenance in the same weeks so that the demand is larger than the available capacity leading to Energy Not Supplied. Since the cost of the mentioned fictitious generator is very large, we are in fact penalizing this maintenance schedule solution.

For each of the mentioned 29 thermal stations we specified the corresponding variable generation cost, the minimum output power, the forced outage rate, the duration in weeks of the corresponding maintenance. As an example, Table I includes the installed capacity, the duration of the maintenance and the forced outage rates.

Apart from these elements, we also specified the number of crews available for each technology, the demand for step $\mathrm{j}$ in each week $t$, the duration of each step as indicated in Section III.A and the energy obtained from the hydro subsystem, from 
the wind parks, from other generation sources and from the interconnections for each step $\mathrm{j}$ and week $\mathrm{t}$. This means that for each step $\mathrm{j}$ and week $\mathrm{t}$ it is possible to obtain the energy to be supplied by the thermal system and according with these values and the generation variable costs it is possible to obtain a dispatch and so to estimate the generation cost of that period.

TABLE I - DATA FOR THE THERMAL GENERATION SYSTEM.

\begin{tabular}{|l|c|c|c|l|c|c|c|}
\hline Type & $\mathrm{P}^{\max }$ & Sk & FOR & Type & $\begin{array}{c}\mathrm{P}^{\max } \\
\text { MW }\end{array}$ & $\begin{array}{c}\text { Sk } \\
\text { week }\end{array}$ & $\begin{array}{l}\text { FOR } \\
\text { week }\end{array}$ \\
\hline Coal1 & 292 & 3 & 3.40 & CCGT8 & 330 & 4 & 4.80 \\
\hline Coal2 & 292 & 3 & 3.40 & Fuel1 & 236 & 6 & 8.50 \\
\hline Coal3 & 292 & 3 & 3.40 & Fuel2 & 236 & 6 & 8.50 \\
\hline Coal4 & 292 & 3 & 3.40 & Fuel3 & 236 & 6 & 8.50 \\
\hline Coa15 & 298 & 3 & 8.70 & Fuel4 & 236 & 6 & 8.50 \\
\hline Coal6 & 298 & 3 & 8.70 & Fuel5 & 118 & 5 & 13.70 \\
\hline Coal7 & 298 & 3 & 8.70 & Fuel6 & 118 & 5 & 13.70 \\
\hline Coal8 & 298 & 3 & 8.70 & Fuel7 & 118 & 5 & 13.70 \\
\hline CCGT1 & 420 & 3 & 1.10 & Fuel8 & 118 & 5 & 13.70 \\
\hline CCGT2 & 420 & 3 & 1.10 & Fuel9 & 118 & 5 & 13.70 \\
\hline CCGT3 & 392 & 3 & 1.10 & Fuel10 & 118 & 5 & 13.70 \\
\hline CCGT4 & 392 & 3 & 1.10 & Fuel11 & 56 & 5 & 11.50 \\
\hline CCGT5 & 392 & 3 & 1.10 & Diesel1 & 82,5 & 2 & 4.30 \\
\hline CCGT6 & 330 & 4 & 4.80 & Diesel2 & 82,5 & 2 & 4.30 \\
\hline CCGT7 & 330 & 4 & 4.80 & ENS & 1000 & -- & - \\
\hline
\end{tabular}

\section{B. Results for Simulation 1}

In the first simulation we admitted that all units were available in all weeks during the year, that is $i_{k t}=1$ for every $\mathrm{k}$ and $t$. On the other hand there is no preference on particular weeks to subject units to the maintenance actions. This means that in this first simulation we admitted that $t_{\bar{k}}=1$ and that $t_{k}^{+}=52$. In order to run the Simulated Annealing algorithm we specified that the number of iterations to run at the same temperature level was 200 , that the number of worse solutions before convergence was 300 and the temperature-lowering coefficient was set at 0.95 .

The first solution was obtained by a random procedure and the corresponding value of the evaluation function was larger than $31.4 .10^{9} €$. This large value is very much determined by penalties on violated constraints. The graph in Figure 2 illustrates the evolution of the evaluation function for the current solution and for the so far best identified solution. After the first thousand iterations the evaluation function decreased to about $1.4 .10^{9} €$, that is decreased by about $30.10^{9}$ $€$. This indicates that it was possible to identify solutions not violating any constraint and also completely supplying the demand without using the fictitious generator representing Energy Not Supplied. In this case, the simulation ends after 14130 iterations and the temperature is lowered till 0.243 . The final schedule displays a relatively large concentration of maintenance actions in the period from week 14 to 23 . This is due to fact that in these weeks the energy demand to be supplied by thermal stations is more reduced because there are larger inputs from hydro stations and wind parks.

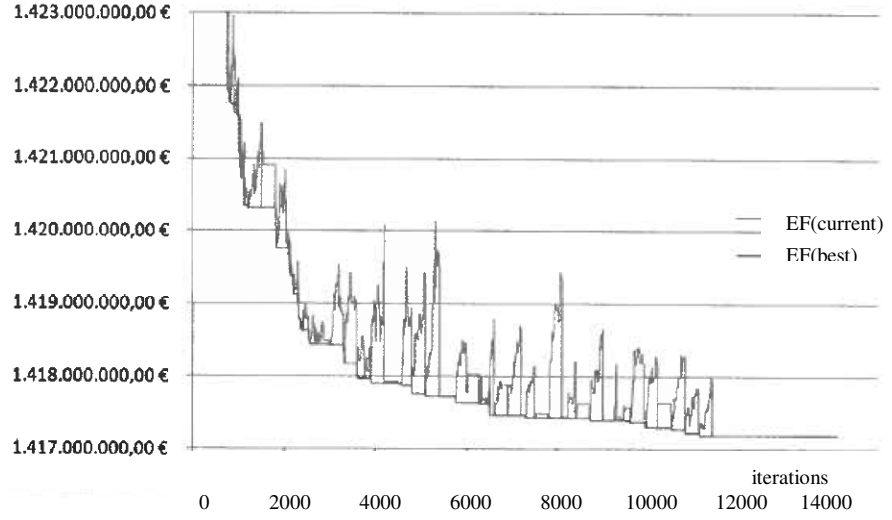

Fig. 2. Evolution of the Evaluation Function along the iterative process.

\section{Results for Simulation 2}

In the second simulation we admitted that some units were not available in some weeks. For instance, one of the CCGT units was not available from week 45 to 52 , one coal station was not available from week 45 to 52 , one fuel station was not available from week 16 to 22 and another fuel station was not available from week 30 to 37 . Apart from that, for some stations we specified values for $t_{k}^{-}$and for $t_{k}^{+}$different from 1 and from 52, respectively. This means that we specified preferences for the scheduling of their maintenance actions. For instance, for one CCGT we specified $t_{k}^{+}=20$, for another CCGT unit we specified $t_{k}^{+}=30$, for one fuel unit we specified $t \bar{k}=30$ and $t_{k}^{+}=50$ and for another fuel station we specified $t_{\bar{k}}=20$ and $t_{k}^{+}=40$. The graph in Figure 3 shows the evolution of the Evaluation Function after the first two thousand iterations. As in Simulation 1, in the beginning the value of the Evaluation Function is very large due to penalties on violated constraints and also due to the fact that there is Energy Not Supplied. As the process goes on, constraint violations are eliminated and Energy Not Supplied comes to zero. The iterative process ends after 23997 iterations and the temperature is lowered to 0.110 . Finally, Table II shows the final maintenance schedule for the 52 weeks of the year. This schedule still shows a large concentration of maintenance actions from week 14 to 25 because the demand values and the inputs from hydro and wind parks are the same ones that were used in the first simulation.

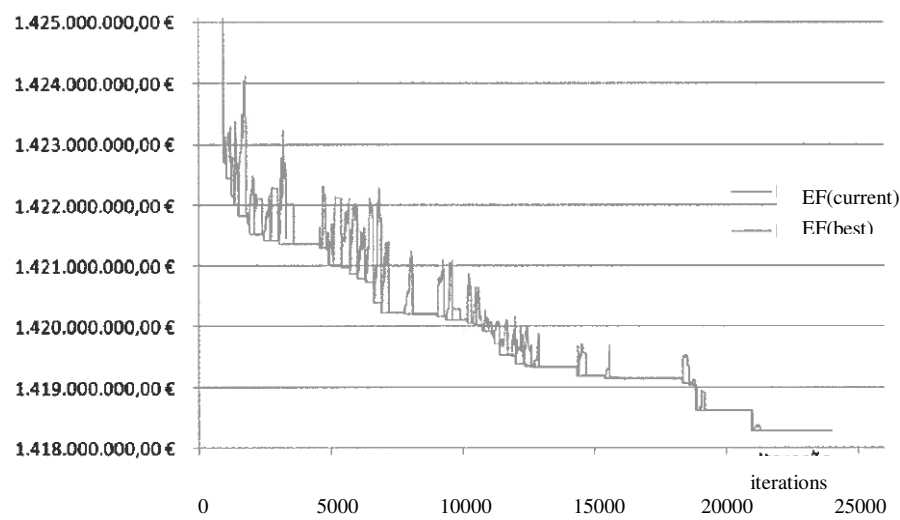

Fig. 3. Evolution of the Evaluation Function along the iterative process. 
Table II - Final maintenance schedule obtained for Simulation 2 (Part 1)

\begin{tabular}{|c|c|c|c|c|c|c|c|c|c|c|c|c|c|c|c|c|c|c|c|c|c|c|c|c|c|c|}
\hline & 1 & 2 & 3 & 4 & 5 & 6 & 7 & 8 & 9 & 10 & 11 & 12 & 13 & 14 & 15 & 16 & 17 & 18 & 19 & 20 & 21 & 22 & 23 & 24 & 25 & 26 \\
\hline $\begin{array}{l}\text { Coal1 } \\
\end{array}$ & & & & & & & & & & & & & & & & & & & & & & & & & & \\
\hline Coal2 & & & & & & & & & & & & & & & & & & & & & & & & & & \\
\hline Coal3 & & & & & & & & & & & & & & & & & & & & & & & & & & \\
\hline $\begin{array}{l}\text { Coal4 } \\
\end{array}$ & & & & & & & & & & & & & & & 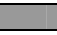 & 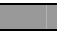 & & & & & & & & & & \\
\hline Coal5 & & & & & & & & & & & & & & & & & & & & & & & & & & \\
\hline $\begin{array}{l}\text { Coal6 } \\
\end{array}$ & & & & & & & & & & & & & & & & & & & & & & & & & & \\
\hline Coal7 & & & & & & & & & & & & & & & & & & & & & & & & & & \\
\hline $\begin{array}{ll}\text { Coal8 } \\
\end{array}$ & & & & & & & & & & & & & & & & & & & & & & & & & & \\
\hline CCGT1 & & & & & & & & & & & & & & & & & & & & & & & & & & \\
\hline CCGT2 & & & & & & & & & & & & & & & & & & & & & & & & & & \\
\hline CCGT3 & & & & & & & & & & & & & & & & & & & & & & & & & & \\
\hline CCGT4 & & & & & & & & & & & & & & & & & & & & & & & & & & \\
\hline CCGT5 & & & & & & & & & & & & & & & & & & & & & & & & & & \\
\hline CCGT6 & & & & & & & & & & & & & & & & & & & & & & & & & & \\
\hline CCGT7 & & & & & & & & & & & & & & & & & & & & & & & & & & \\
\hline CCGT8 & & & & & & & & & & & & & & & & & & & & & & & & & & \\
\hline Fuel1 & & & & & & & & & & & & & & & & & & & & & & & & & & \\
\hline Fuel2 & & & & & & & & & & & & & & & & & & & & & & & & & & \\
\hline Fuel3 & & & & & & & & & & & & & & & & & & & & & & & & & & \\
\hline Fuel4 & & & & & & & & & & & & & & & & & & & & & & & & & & \\
\hline Fuel5 & & & & & & & & & & & & & & & & & & & & & & & & & & \\
\hline $\begin{array}{l}\text { Fuel6 } \\
\end{array}$ & & & & & & & & & & & & & & & & & & & & & & & & & & \\
\hline Fuel7 & & & & & & & & & & & & & & & & & & & & & & & & & & \\
\hline Fuel8 & & & & & & & & & & & & & & & & & & & & & & & & & & \\
\hline $\begin{array}{l}\text { Fuel9 } \\
\end{array}$ & & & & & & & & & & & & & & & & & & & & & & & & & & \\
\hline $\begin{array}{l}\text { Fuel10 } \\
\end{array}$ & & & & & & & & & & & & & & & & & & & & & & & & & & \\
\hline Fuel11 & & & & & & & & & & & & & & & & & & & & & & & & & & \\
\hline Diesel1 & & & & & & & & & & & & & & & & & & & & & & & & & & \\
\hline$D^{\text {Diesel2 }}$ & & & & & & & & & & & & & & & & & & & & & & & & & & \\
\hline
\end{tabular}

Table II - Final maintenance schedule obtained for simulation 2 (Part 2)

\begin{tabular}{|c|c|c|c|c|c|c|c|c|c|c|c|c|c|c|c|c|c|c|c|c|c|c|c|c|c|c|}
\hline & 27 & 28 & 29 & 30 & 31 & 32 & 33 & 34 & 35 & 36 & 37 & 38 & 39 & 40 & 41 & 42 & 43 & 44 & 45 & 46 & 47 & 48 & 49 & 50 & 51 & 52 \\
\hline Coal1 & & & & & & & & & & & & & & & & & & & & & & & & & & \\
\hline Coal2 & & & & & & & & & & & & & & & & & & & & & & & & & & \\
\hline Coal3 & & & & & & & & & & & & & & & & & & & & & & & & & . & \\
\hline Coal4 & & & & & & & & & & & & & & & & & & & & & & & & & & \\
\hline Coal5 & & & & & & & & & & & & & & & & & & & & & & & & & & \\
\hline Coal6 & & & & & & & & & & & & & & & & & & & & & & & & & & \\
\hline $\begin{array}{l}\text { Coal7 } \\
\end{array}$ & & & & & & & & & & & & & & & & & & & & & & & & & & \\
\hline Coal8 & & & & & & & & & & & & & & & & & & & & & & & & & & \\
\hline CCGT1 & & & & & & & & & & & & & & & & & & & & & & & & & & \\
\hline CCGT2 & & & & & & & & & & & & & & & & & & & & & & & & & & \\
\hline CCGT3 & & & & & & & & & & & & & & & & & & & & & & & & & & \\
\hline CCGT4 & & & & & & & & & & & & & & & & & & & & & & & & & & \\
\hline CCGT5 & & & & & & & & & & & & & & & & & & & & & & & & & & \\
\hline CCGT6 & & & & & & & & & & & & & & & & & & & & & & & & & & \\
\hline CCGT7 & & & & & & & & & & & & & & & & & & & & & & & & & & \\
\hline CCGT8 & & & & & & & & & & & & & & & & & & & & & & & & & & \\
\hline Fuel1 & & & & & & & & & & & & & & & & & & & & & & & & & & \\
\hline Fuel2 & & & & & & & & & & & & & & & & & & & & & & & & & & \\
\hline Fuel3 & & & & & & & & & & & & & & & & & & & & & & & & & & \\
\hline Fuel4 & & & & & & & & & & & & & & & & & & & & & & & & & & \\
\hline Fuel5 & & & & & & & & & & & & & & & & & & & & & & & & & & \\
\hline Fuel6 & & & & & & & & & & & & & & & & & & & & & & & & & & \\
\hline Fuel7 & & & & & & & & & & & & & & & & & & & & & & & & & & \\
\hline Fuel8 & & & & & & & & & & & & & & & & & & & & & & & & & & \\
\hline Fuel9 & & & & & & & & & & & & & & & & & & & & & & & & & & \\
\hline Fuel10 & & & & & & & & & & & & & & & & & & & & & & & & & & \\
\hline Fuel11 & & & & & & & & & & & & & & & & & & & & & & & & & & \\
\hline Diesel1 & & & & & & & & & & & & & & & & & & & & & & & & & & \\
\hline Diesel2 & & & & & & & & & & & & & & & & & & & & & & & & & & \\
\hline
\end{tabular}

\section{CONCLUSIONS}

In this paper we addressed the problem of building good quality generator maintenance schedules given the relevance of this topic in the context of the advent of competition in the electricity sector. This is a complex optimization problem formulated as a mixed-integer problem for which we applied Simulated Annealing, given its abilities to address combinatorial problems and its easiness of implementation. The formulation minimizes the generation cost along the maintenance planning horizon and it implicitly includes a reliability measure when penalizing non zero values of Energy Not Supplied. The problem integrates constraints related with the continuity of the maintenance actions, with the limited number of crews for some generation technologies and with the preferences of the Decision Maker to locate some maintenance actions along the year. The Simulated Annealing application shows good performance and it is able to produce good maintenance schedules with short computation times.

\section{ACKNOWLEDGMENT}

The second author thanks EDP Produção SA for having provided guidance and data to conduct this research.

\section{REFERENCES}

[1] H. H. Zurn, V. H. Quintana, "Several Objective Criteria for Optimal Generator Preventive Maintenance Scheduling", IEEE Transaction on Power, Apparatus and Systems, Vol. PAS-96, no. 3, May/June 1977.

[2] L. F. Escudero, "On Maintenance Scheduling of Production Units", European Journal of Operational Research, Vol. 9, no. 3, March 1982. 
[3] B. Krajl, N. Rajakovic, "Multiobjective Programming in Power System Optimization: New Approach to Generator Maintenance Scheduling", Electrical Power \& Energy Systems, Vol. 16, no. 4, August 1994.

[4] R. Mukerji, H. M. Merrill, B. W. Erickson, J. H. Parker, R. E. Friedman, "Power Plant Maintenance Scheduling: Optimizing Economics and Reliability", IEEE Transaction on Power Systems, Vol. 6, no. 2, May 1991.

[5] M. K. C. Marwali, S. M. Shahidehpour, "A Probabilistic Approach to Generation Maintenance Scheduler with Network Constraints", Electrical Power \& Energy Systems, Vol. 21, no. 8, November 1999.

[6] T. Satoh, K. Nara, "Maintenance Scheduling by Using Simulated Annealing Method", IEEE Transactions on Power Systems, Vol. 6, no. 2, May 1991.

[7] K. P. Dahal, J. R. McDonald, "Generator Maintenance Scheduling of Electric Power Systems Using Genetic Algorithms and Integer Representation", in Proceedings of the $2^{\text {nd }}$ Int. Conf. on Genetic Algorithms in Engineering Systems, Glasgow, UK, September 1997.

[8] H. Kim, K. Nara, M. Gen, "A Method for Maintenance Scheduling Using GA Combined with SA", Computers \& Industrial Engineering, Vol. 27, no. 1-4, September 1994.

[9] K. P. Dahal, N. Chakpitak, "Generator Maintenance Scheduling in Power Systems Using Metaheuristic-Based Hybrid Approaches", Electric Power Systems Research, Vol. 77, no. 7. May 2007.

[10] S.-J. Huang, "A Genetic-Evolved Fuzzy System for Maintenance Scheduling of Generation Units", Electrical Power \& Energy Systems, Vol. 20, no. 3, June 1998

[11] K. P. Dahal, C. J. Aldridge, J. R. McDonald, "Generator Maintenance Scheduling Using a Genetic Algorithm with a Fuzzy Evaluation Function", Fuzzy Sets and Systems, Vol. 102, no. 1, February 1999.

[12] M. Y. El-Sharkh, A. A. El-Keib, H. Chen, "A Fuzzy Evolutionary Programming-Based Solution Methodology for Security-Constrained Generation Maintenance Scheduling", Electric Power Systems Research, Vol. 67, no. 1, October 2003.

[13] E. K. Burke, A. J. Smith, "A Multi-Stage Approach for the Thermal Generator Maintenance Scheduling Problem", Proceedings of the 1999 Congress on Evolutionary Computation, CEC'99, Vol. 2, Washington DC, USA, July 1999.

[14] I. El-Amin, S. Duffuaa, M. Abbas, "A Tabu Search Algorithm for Maintenance Scheduling of Generating Units", Electric Power Systems Research, Vol. 54, no. 2, May 2000.

[15] E. K. Burke, A. J. Smith, "Hybrid Evolutionary Techniques for the Maintenance Scheduling Problem", IEEE Transactions on Power Systems, Vol. 15, no. 1, February 2000.

[16] K. P. Dahal, S. J. Galloway, "Evolutionary Generator Maintenance Scheduling in Power Systems", Studies in Computational Intelligence, Vol. 49, 2007.
[17] J.-H. Kim, J.-B. Park, J.-K. Park, Y.-H. Chun, "Generating Unit Maintenance Scheduling Under Competitive Market Environment", Electrical Power \& Energy Systems, Vol. 27, no. 3, March 2005.

[18] S. Kirkpatrick, C. D. Gelatt, M. P. Vecchi, "Optimization by Simulated Annealing", Science, vol. 220, no. 4598, pp. 671 - 680, 1983.

[19] E. Aarts, J. Korst, Simulated Annealing and Boltzman Machines, New York, John Wiley \& Sons, 1990.

\section{BIOGRAPHIES}

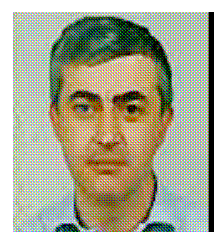

João Tomé Saraiva was born in Porto, Portugal in 1962. In 1987, 1993 and 2002 he got his MSc, PhD, and Agregado degrees in Electrical and Computer Engineering from the Faculdade de Engenharia da Universidade do Porto, FEUP, where he is currently Professor. In 1985 he joined INESC Porto - a private research institute - where he is head researcher and collaborated in several projects namely in consultancy contracts established with the Portuguese Electricity Regulatory Agency.

Marcelo Leandro Pereira enrolled in Faculdade de Engenharia da Universidade do Porto, FEUP, in September 2003 and concluded the MSc in Electrotechnic and Computer Engineering, Major in Energy, in March 2009. His thesis addressed the optimization of the preventive maintenance scheduling of generators. This paper reports the most relevant results obtained with his work.

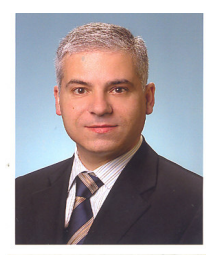

Virgílio Torrado Mendes was born in Porto, Portugal in 1959. In 1978 got the Bachelor of Mechanics Engineering from Instituto Superior de Engenharia do Porto and in 1981 he got his graduation in Mechanical Engineering from Faculdade de Engenharia da Universidade do Porto, FEUP. In 1985 he joined EDP and since 1989 he has worked in expansion studies of power generation systems in the company. He was call as an invited expert by International Energy Atomic Agency for training courses in Mexico and Egypt.

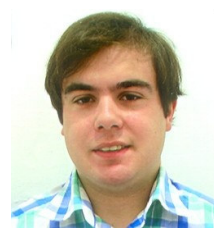

José Carlos Sousa was born in Porto, Portugal in 1982. In 2005 and 2007 he got the licenciate and the MSc degrees from the Faculdade de Engenharia da Universidade do Porto, FEUP. Since 2006 he works in EDP Produção in the Planning and Control Department and since 2008 he is $\mathrm{PhD}$ student at FEUP. 